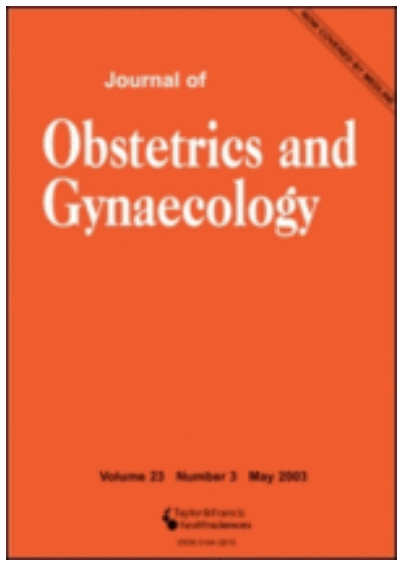

\title{
Changes in vascular function and autonomic balance during the first trimester of pregnancy and its relationship with the new-born weight
}

\begin{tabular}{|r|l|}
\hline Journal: & Journal of Obstetrics and Gynaecology \\
\hline Manuscript ID & CJOG-2019-0917.R1 \\
\hline Manuscript Type: & Research Article \\
\hline Author: & 03-Jan-2021 \\
\hline Complete List of Authors: & $\begin{array}{l}\text { Benvenuto, Silvia; Instituto de Maternidad Nuestra Señora de las } \\
\text { Mercedes -Sistema Provincial de Salud (SIPROSA) } \\
\text { Joo Turoni, Claudio; Depto. Biomédico - Or Fisiología, Facultad de } \\
\text { Medicina - UNT; INSIBO -CONICET } \\
\text { Maranon, Rodrigo; Depto. Biomédico - Or Fisiología, Facultad de } \\
\text { Medicina - UNT; INSIBO -CONICET } \\
\text { Chahla, Rossana; Instituto de Maternidad Nuestra Señora de las } \\
\text { Mercedes -Sistema Provincial de Salud (SIPROSA) } \\
\text { Peral de Bruno, María; Depto. Biomédico - Or Fisiología, Facultad de } \\
\text { Medicina - UNT; INSIBO -CONICET }\end{array}$ \\
\hline Keywords: & $\begin{array}{l}\text { Arterial stiffness, heart rate variability, sympathetic system } \\
\text { parasympathetic system, weight of the newborn }\end{array}$ \\
\hline & \\
\hline
\end{tabular}

\section{SCHOLARONE" Manuscripts}




\title{
Changes in vascular function and autonomic balance during the first trimester of pregnancy and its relationship with the new-born weight
}

\author{
Silvia Benvenuto ${ }^{\mathrm{a}}$, Claudio Joo Turoni ${ }^{\mathrm{b}}$, Rodrigo O Marañón ${ }^{\mathrm{b}}$, Rossana \\ Chahla ${ }^{a}$, María Peral de Bruno ${ }^{\mathrm{b} *}$ \\ a Instituto de Maternidad Nuestra Señora de las Mercedes -Sistema Provincial de Salud \\ (SIPROSA)- Tucumán - Argentina ; ${ }^{b}$ Departamento Biomédico, Instituto de Fisiología, \\ Facultad de Medicina - UNT; INSIBO -CONICET - Tucumán - Argentina
}

*Corresponding author

Dr. María Peral de Bruno

Address: Centro Herrera - Av. Roca 1900 - Tucumán (4000) Argentina

Phone: 00543816787486

E-mail: mperal0150@gmail.com

Running head: Vascular Function in the First Trimester of Pregnancy and Born-weight

Funding: This study was funded by the National Scientific and Technical Research Council (CONICET) 


\title{
Changes in vascular function and autonomic balance during the first trimester of pregnancy and its relationship with the new-born weight
}

\begin{abstract}
This study aimed to evaluate vascular function changes and autonomic balance during the first trimester of pregnancy and its relationship with the new-born weight. This prospective study performed in pregnant women (PG) and after delivery (Not pregnant: NPG) evaluated the endothelial function (EF) and arterial stiffness (AS) by a non-invasive method. We evaluated the heart rate variability (HRV), parasympathetic (PNS), sympathetic (SNS) indexes by electrocardiogram (5 min), and the urinary nitrite excretion (NOx). PG increased EF and NOx and decreased AS and HRV. PG decreased the PNS index and augmented the SNS index. The new-born weight positively correlated with the PNS index (Pearson r: 0.4291; $\mathrm{p}<0.05), \mathrm{NOx}, \mathrm{HRV}$, and negatively correlated with AS. In summary, in pregnancy, although hemodynamically, the SNS activation plays a compensatory role, the low rates of PNS inhibition are essential to ensure normal fetal growth.
\end{abstract}

Keywords: Arterial stiffness; heart rate variability; sympathetic system parasympathetic system; the new-borns weight. 


\title{
Impact Statement
}

What is already known on this subject? In pregnancy, there are adaptive physiological changes in the cardiovascular system that include increases of EF and decreases AS with an SNS activation. The study of HRV lets to predict the SNS and PNS balance and how they affect blood pressure and vascular function.

What the results of this study add? Although it is known that SNS activation plays a compensatory role in normal pregnancy, this study adds the critical role of PNS. Early in pregnancy, the low rates of PNS inhibition are essential to ensure normal fetal growth.

\begin{abstract}
What the implications are of these findings for clinical practice and/or further research? The present results show a potential predictive value of SNS and PNS activity early in pregnancy. It will provide valuable information not only on the pregnant woman's vascular function but also on the new-born weight.
\end{abstract}




\section{Introduction}

In pregnancy, there are adaptive changes in the cardiovascular system (Alquezar et al. 2018) that are accompanied by an expansion of plasma volume (Rodger et al. 2015), declining in blood pressure, systemic vasodilation and an increase in heart rate (HR) (Walters et al. 1969; Fisher et al. 2002). Furthermore, pregnancy modifies the arterial tone, which is regulated by the endothelial function (EF) through the nitric oxide (NO) (Furchgott et al. 1980). The EF is early implicated in the pathophysiology of cardiovascular diseases (Dzau 2004). Also, vascular compliance alterations have been associated with cardiovascular events (Laurent et al. 2001). Therefore, EF and arterial stiffness (AS) could be independent prognostic indicators of cardiovascular damage (Laurent et al. 2006).

EF and AS could be evaluated through a non-invasive method (Kuvin et al. 2003; Itzhaki et al. 2005). Using this methodology, we found a decreased EF without AS alteration in obese adolescents and low birth weight children (Joo Turoni et al. 2013 and 2016). Torrado observed an improved EF and aortic stiffness in pregnancy (Torrado et al. 2015) and Savvidou reported that increased AS at 22-24 weeks of gestation predates the pre-eclampsia development (Savvidou et al. 2011). If normal pregnancy plays a role in EF and AS needs to be investigated.

The beat-to-beat HR variability (HRV) has been used to study the sympathetic (SNS) and parasympathetic (PNS) systems balance. Evidence shows that these systems' changes modify the HRV (Lombardi et al. 1996; Nunan et al. 2010). Also, the low birth weight in children decreases the HRV, predicting morbidity and mortality (Rakow et al. 2013, Wulsin et al. 2015). 
In pregnancy, hypertension (Ferrazzani et al. 2011) and diabetes (Vambergue et al. 2011) modify the new-born weight. Besides, Everett reported that new-born weight is related to maternal HR (Everett et al., 2013).

Therefore, for all mentioned above, we aimed to evaluate vascular function changes and autonomic balance during the first trimester of pregnancy and its relationship with the new-born weight.

\section{Material and Methods}

A prospective cohort study in women during the first trimester of normal pregnancy (Pregnant: PG) and after 9 to 12 months of delivery (Not pregnant: NPG) was performed. Women were enrolled in the Instituto de Maternidad Nuestra Señora de las Mercedes (Tucumán, Argentina). Exclusion criteria: cardiovascular disease, hypertension, diabetes, glucose intolerance, and smoking; multiple pregnancies or clinical report of infections in the current pregnancy, altered uterine doppler and preterm delivery. In NPG, women were studied when presenting a menstrual cycle return. Since it was established that vascular function is improved in the follicular phase (Williams et al. 2001), the studies in NPG were performed 2-3 days after the end of the menstrual cycle. Also, weight, height, and gestational age of the new-borns were recorded from the clinical history.

\section{Ethical procedure}

The Ethics Research Committee of the Research Department of the Health Ministry of Tucumán approved all procedures (Protocol \#89). Besides, all participants provided oral and written informed consent to participate before any procedure.

\section{Hemodynamic variables}


Systolic (SBP), diastolic (DBP), and mean blood pressure (MBP), and heart rate (HR) were measured.

\section{Endothelial Function Assessment}

EF was evaluated through plethysmography, as we previously described (Joo Turoni et al. 2013 and 2016). To record and measure the waveforms, we used a plethysmograph connected to an electrocardiograph (Dong Jiang 32A; Chine). Also, to standardize the chart recording, the equipment was calibrated as follow: $1 \mathrm{~mm}=0.1 \mathrm{mV}$ velocity: 25 $\mathrm{mm} / \mathrm{sec}$. The hyperaemic response was obtained by flow-mediated vasodilatation (Joo Turoni et al. 2013). Briefly, a photoelectric transducer was placed on the index finger of the left hand. Previously to the recording phase, women hold their breath for 10 seconds (pre-occlusion phase). Then, the sphygmomanometer cuff was insufflated until 50 $\mathrm{mmHg}$ above SBP by 5 minutes (occlusion phase). Subsequently, the sphygmomanometer cuff was deflated, and after 2 minutes, another 10 seconds of apnea was required (post-occlusion phase). The record obtained was scanned to measure the pulse wave amplitude (valley/peak size) using Image J 1.52a (Maryland, USA). Ten consecutive waves were averaged from each phase to compare pre-occlusion vs postocclusion phases.

\section{Arterial Stiffness Assessment}

The AS index was calculated through the plethysmography system (Joo Turoni et al. 2013). The procedures were similar to those described above to evaluate EF. A graphic record of ten pulse-waves was obtained with a simultaneous record of the electrocardiogram. Registers obtained from each woman were scanned to determine the AS index. Image J 1.52a software was used to calculate the amplitude values $\left[\left(a^{*} 100 / b\right.\right.$, where $a=$ maximal systolic peak amplitude $(\mathrm{mm})$ and $b=$ maximal diastolic peak amplitude (mm)]. 


\section{HRV and Autonomic Function}

A continuous electrocardiogram record (5 minutes) at rest sitting was acquired (Taurus Touch; JotaTec; Argentina) connected to a computer. The distance between R waves of the consecutive beats (RR interval) of the complete DII lead record was obtained. The HRV was analysed with Kubios HRV 3.1 software (Kuopio, Finland). In the time domain, the mean RR interval, and the percentage of consecutive beats that differ by more than $50 \mathrm{~ms}$ (pNN50), were calculated. Similarly, the standard deviation of the time that separates two successive beats (SDNN) and the HR were analysed. In the frequency domain, the low, high, and total frequency powers (LF, HF, and T) and LF/HF ratio were measured. Also, Kubios HRV 3.1 software made a non-linear geometric analysis using the Poincaré plot scatter. It graphs the relationship RRn (xaxis) and $\mathrm{RRn}+1$ (y-axis) which let calculate the transverse axis (SD1), the longitudinal axis (SD2), and the SD2/SD1 ratio. Also, it calculates the mean deviation from typical values of PNS and SNS indexes (Nunan et al. 2010).

\section{Urinary Nitrites}

Urinary nitrite excretion (NOx) is a non-traumatic indicator of $\mathrm{NO}$ bioconversion/bioavailability (Elli et al. 2005; Vieira de Oliveira et al. 2016). The NOx was measured by the Griess reaction (Marañón et al. 2014) in a fasting urine sample.

\section{Statistical Analysis}

The data were expressed as media \pm standard error. $\mathrm{P}<0.05$ values were considered statistically significant. The Statistical 5.0 and Graph-Pad Prism 5.02 software were used for statistical analysis and graphs. A paired Student t-test or Pearson correlation coefficient were used when necessary. 


\section{Results}

In PG, the women's age was $28.7 \pm 0.8$ years, and the gestational age was $10 \pm 0.2$ weeks. Although in PG the blood pressure was maintained into the normal range, a significant decrease of SBP (PG: 104 \pm 2 mmHg; vs NPG: $113 \pm 3 \mathrm{mmHg} ; \mathrm{n}=25 ; \mathrm{p}<0.001$ ) and DBP (PG: $69 \pm 2 \mathrm{mmHg}$; vs NPG: $74 \pm 2 \mathrm{mmHg}$; $\mathrm{n}=25$; $<<0.001$ ) were observed. Likewise, a decrease in pulse pressure (PG: $36 \pm 2 \mathrm{mmHg}$; vs NPG: $40 \pm 2 \mathrm{mmHg} ; \mathrm{n}=25 ; \mathrm{p}<0.05$ ) and in MBP in PG $(81 \pm 2 \mathrm{mmHg}$ vs NPG $(87 \pm 2 \mathrm{mmHg} ; \mathrm{n}=25 ; \mathrm{p}<0.001)$ was observed.

\section{Endothelial Function and Arterial Stiffness}

The endothelial-dependent response was markedly increased in PG (PG: $102 \pm 19 \%$ vs NPG: $24 \pm 4 \% ; n=25 ; \mathrm{p}<0.001$ ), whereas the AS index was decreased (PG: $43 \pm 2 \%$ vs NPG: $52 \pm 2 \% ; \mathrm{n}=25 ; \mathrm{p}<0.001)$. The women's age was not correlated with the endothelial-dependent response (PG: Pearson r: 0.2057; IC95\%: -0.2063 to 0.5558 ; p: NS and NPG: 0.06080; IC95\%: -0.3427 to 0.4453 ; p: NS), and with AS index (PG: Pearson r: -0.03598 ; IC95\%: -0.4252 to 0.3644 ; p: NS, and NPG: 0.03214 ; IC95\%: 0.3678 to $0.4220 ; \mathrm{p}$ : NS).

\section{HRV and Autonomic Function}

The HRV analysis in the time and frequency domains is shown in Table 1. In PG, HR was increased, and the RR interval, pNN50, and SDNN were decreased compared to NPG. In the frequency domain, PG showed a reduction in LF, HF, and total power, while LF/HF was not modified. The non-linear geometric analysis in PG shows a decreased in both SD1 (PG: $27 \pm 2$ ms vs NPG: 41 \pm 3 ; p $<0.001$ ) and SD2 (PG: $38 \pm 3$ ms vs NPG: $60 \pm 5 ; \mathrm{p}<0.001$ ). Nevertheless, the SD2/SD1 ratio was not modified (PG: $1.4 \pm 0.1$ vs NPG: $1.5 \pm 0.1$; p: NS).

Figure 1A shows typical reports of PNS and SNS indexes obtained in PG (top) and NPG (bottom). The index averages are shown in Figure 1B. In PG, the PNS index 
was decreased, whereas the SNS index was increased, but the decrease in the PNS index $(-45 \pm 7 \%)$ was lower than the increase in SNS index $(+282 \pm 120 \% ; \mathrm{p}<0.05)$.

\section{Urinary Nitrites}

The NOx was increased in PG (PG: $4.5 \pm 0.5$ uM/L vs NPG: $1.4 \pm 0.1 ; p<0.001$ ) and positively correlated only with PNS index (Pearson r: 0.5748; IC95\%: 0.2323 to 0.7904; $\mathrm{p}<0.01$ ). In NPG, none correlation between NOx and PNS or SNS indexes were found (PNS vs NOx: Pearson r: -0.1196; IC95\%: -0.4916 to 0.2893; p: NS; and SNS vs NOx: Pearson r: 0.01835; IC95\%: -0.3796 to 0.4106 ; p: NS).

Correlation between new-borns weight, height, and gestational age and the EF, AS, NOx, and HRV parameters

The mean body weight was $3,274 \pm 59 \mathrm{~g}$, and the mean height was $49 \pm 1 \mathrm{~cm}$ (considered within normal range). Similarly, as occurred with EF and AS, the women's age did not correlate with other parameters.

The EF correlates with neither weight nor height of the new-born in both PG and NPG. However, only in PG, AS index was negatively correlated with new-borns weight (Figure 2A). In PG, the NOx was positively correlated with the new-born weight (Figure 2B) and the new-born height (Pearson r: 0.3995; IC95\%: 0.005029 to 0.6863 ; $\mathrm{p}<0.05)$.

In $\mathrm{PG}$, in the time domain, only pNN50 showed a positive correlation with the new-born weight (Figure 3A). Contrary to pNN50, the HR, RR interval, and SDNN did not present any correlation (data not showed). In PG, in the frequency domain, HF was positively correlated with the new-born weight (Figure 3B). However, in LF, total power, and LF/HF ratio, none correlation was observed (data not showed). Besides, in the non-linear geometric analysis, SD1 was positively correlated with the new-born weight (Pearson r: 0.4234; 95\%CI: 0.03391 to 0.7013 ; Figure $3 \mathrm{C} ; \mathrm{p}<0.05$ ). No 
correlation was observed in the SD2 and SD2/SD1 ratio (data not showed). In NPG, in the time domain, frequency domain, and non-linear geometric analysis variables, none correlation was observed (data not showed).

In PG, PNS index was positively correlated with the new-borns weight (Pearson r: 0.4291; IC95\%: -0.04078 to 0.7048; $\mathrm{p}<0.05$ ) and height (Pearson R: 0.4243; IC95\%: 0.03500 to $0.7019 ; \mathrm{p}<0.05)$. SNS index showed no correlation with the same factors (Weight: Pearson r: -0.3415; IC95\%: -0.6491 to 0.06212; p: NS and Height: Pearson R: -0.1266 ; IC95\%: -0.4970 to 0.2828 ; p: NS). In NPG, there is no correlation between PNS and SNS indexes with weight or height (data not showed).

\section{Discussion}

The main findings of the present study are: 1) In early pregnancy there is an increase in EF and a decreased in AS and HRV, 2) These changes are accompanied by an increased SNS index and a decreased PNS index, and 3) PNS index was positively correlated with the new-born weight.

During the first trimester of pregnancy, there is a decreased blood pressure due to a fall in the peripheric vascular resistance through dependent-endothelial vasodilation (Leiva et al. 2016). In our study, PG showed a strong EF which decreases after delivery (NPG) with an increased NO release supporting the fact that in pregnancy, the EF is mainly NO-dependent (Ramsay et al. 2003, Tanaka et al. 2015).

Healthy pregnancy enhances EF (Ramsay et al. 2003; Boeldt DS and Bird IM 2017) and reduces AS during all adaptation processes (Mahendru et al. 2014). In PG, we found a decrease in AS, which was described by other authors, as well (Macedo et al. 2008). It could be due to smooth muscle tone or vascular remodelling in different vascular beds (Edouard DA et al. 1998) where the extravillous trophoblast cells mediate 
the uterine decidual spiral arterioles remodelling (Chen et al. 2012). So, the changes in $\mathrm{EF}$ and $\mathrm{AS}$ in the peripheric beds would be involved in the decreased BP at this stage (Mahendru et al. 2014).

The HRV has been used to study the sympathetic and parasympathetic system's balance. Although different studies indicate that the fetal environment plays an essential role in developing cardiometabolic risk later in life, few studies of the impact of autonomic balance and intrauterine growth have been performed. Furthermore, some of its results showed conflicting data (Lahiri et al. 2008).

In our study, we found a decreased HRV in PG. In the time domain, quantifying the amount of HRV, we observed that HR, RR interval, pNN50, and SDDN were reduced (Table 1). Interestingly, only pNN50 was positively correlated with the newborn weight. As pNN50 is an indicator of vagal tone (Koenig et al. 2015), it suggests that the degree of vagal tone inhibition during early pregnancy is related to the newborn weight in the child after delivery. On the other hand, decreased SDNN is an indicator of increased SNS activity (Tarvainen et al. 2014). Since the evidence showed that in pregnancy, the sympathetic activity is increased, it is not surprising that SDNN was reduced. In the frequency domain, which calculates the amount of signal energy within each component band, LF, HF, and total power were also decreased (Table 1).

HF is a specific PNS indicator, whereas LF has associated with both SNS and PNS activity (Malik et al. 1993). It also indicates the role of the inhibition of vagal tone on the new-born weight. Regarding the changes in the non-linear geometric analysis, SD1 (an indicator of PNS activity) and SD2 (inverse SNS activity indicator) were decreased in PG (Kamen et al. 1996; Toichi et al. 1997). Also, PG showed a modified SNS/PNS balance. Recently, Iacobaeus demonstrated that pregnancy produces a simultaneous cardiac and arterial adaptation associated with autonomic balance 
(Iacobaeus et al. 2018). Here, we found a higher increase in the SNS index, where the sympathetic system maintains maternal blood flow through increased minute volume. Therefore, both branches (SNS and PNS) play a role in the hemodynamic regulation during early pregnancy.

In PG, we found that the new-born weight was associated with AS, NOx, and HRV. Everett demonstrated that the birth weight was correlated to the HR; however, these findings were in the mid-trimester maternal, and the group of the domain that characterizes the HRV components was not evaluated (Everett et al. 2013).

In children, HRV is negatively correlated with birth weight (Rakow et al. 2013). Also, in postconceptional age, the HRV is related to intrauterine maturation of the autonomic cardio regulatory activity (Sahni et al. 2000). In this sense, we hypothesize that PNS regulation plays a vital role in the mother-child binomial hemodynamic. This hypothesis is supported because only PNS index correlates with the new-born weight. Furthermore, the new-born body weight was positively correlated with HF and SD1, PNS activity indicators (Malik M et al. 1993; Kamen et al. 1996). The NOx positive correlation with the new-born weight could be due to the PNS effect on the NO bioavailability (Li et al. 2016).

In summary, our study shows that the parasympathetic branch inhibition would be responsible for hemodynamic regulation during the first trimester of pregnancy. It is essential to ensure normal foetal growth. In the counterregulatory PNS/SNS balance, an increase in NO release and a decrease in the maternal peripheral vascular resistance may are implicated. Therefore, the evaluation of the PNS index may help as a clinical parameter in order to evaluate the hemodynamic control during early pregnancy and predicts the new-born weight. 


\section{All authors have no conflicts of interests to declare}

\section{References}

Alquezar T et al. 2018. Pregnancy as an early stress test for cardiovascular and kidney disease diagnosis, Pregnancy Hypertension 12: 169-173.

Boeldt DS and Bird IM. Vascular Adaptation in Pregnancy and Endothelial Dysfunction in Preeclampsia. J Endocrinol. 2017 Jan; 232(1): R27-R44.

Chen J; Sheehan P; Brennecke S; Keogh. 2012. Vessel remodelling, pregnancy hormones, and extravillous trophoblast function. Molecular and Cellular Endocrinology 349: $138-144$

Dzau V. 2004. Markers of Malign Across the Cardiovascular Continuum: Interpretation and Application. Circulation 109: IV1-IV2.

Edouard DA et al. 1998. Venous and arterial behaviour during normal pregnancy. Am J Physiol; 274:1605-1612.

Elli $\mathrm{M}$ et al. 2005. Plasma and urine nitric oxide levels in healthy Turkish children. Pediatr Nephrol 20: 1605-1609.

Everett T, Mahendru A, McEniery C, Wilkinson I, Lees C. 2013. Mid-trimester maternal heart rate is related to neonatal birth weight. J Matern Fetal Neonatal Med 26: $1082-1085$.

Ferrazzani S et al. 2011. Neonatal outcome in hypertensive disorders of pregnancy. Early Hum Dev 87:445-449.

Fisher C, MacLean M, Morecroft I, et al. 2002. Is the pregnancy hormone relaxin also a vasodilator peptide secreted by the heart? Circulation 106:292-5

Iacobaeus $\mathrm{C}$ et al. 2018. Cardiac function, myocardial mechano-energetic efficiency, and ventricular-arterial coupling in normal pregnancy, J Hypertens 36: 857-866. 
Itzhaki S, Lavie L, Pillar G, Tal G, Lavie P. 2005. Endothelial dysfunction in obstructive sleep apnea measured by peripheral arterial tone response in the finger to reactive hyperaemia. Sleep 28: 594-600.

Joo Turoni $\mathrm{C}$ et al. 2013. Arterial stiffness and endothelial function in obese children and adolescents and its relationship with cardiovascular risk factors. Horm Res Paediatr 80: 281-286.

Joo Turoni $\mathrm{C}$ et al. 2016. Vascular function in children with low birthweight and its relationship with early markers of cardiovascular risk. Horm Res Paediatr 85: 396-405.

Kamen P, Krum H, Tonkin H.1996. Poincaré plots of heart rate variability allows quantitative display of parasympathetic nervous activity in humans. Clinical Science 91: 201-208.

Koenig J et al. 2015. Lowered Parasympathetic Activity in Apparently Healthy Subjects with Self-Reported Symptoms of Pain: Preliminary Results from a Pilot Study. Pain Practice. 15: 314-318.

Kuvin J et al. 2003. Assessment of peripheral vascular endothelial function with finger arterial pulse wave amplitude. Am Heart J 146: 168-174.

Lahiri MK, Kannankeril PJ, Goldberger JJ. Assessment of autonomic function in cardiovascular disease: physiological basis and prognostic implications. J Am Coll Cardiol. 2008; 51: 1725-1733. pmid:18452777.

Laurent S et al. 2001. Aortic Stiffness Is an Independent Predictor of All-Cause and Cardiovascular Mortality in Hypertensive Patients. Hypertension 37: 1236-1241.

Laurent S et al. 2006. European network for non-invasive investigation of large arteries: Expert consensus document on arterial stiffness: methodological issues and clinical applications. Eur Heart J 27: 2588-2605. 
Leiva A et al. 2016. Nitric Oxide is a Central Common Metabolite in Vascular Dysfunction Associated with Diseases of Human Pregnancy. Curr Vasc Pharmacol $14: 237-59$.

Li P et al. 2016. Chronic vagus nerve stimulation attenuates vascular endothelial impairments and reduces the inflammatory profile via inhibition of the NF- $\mathrm{BB}$ signaling pathway in ovariectomized rats. Exp Gerontol 74: 43-55.

Lombardi F, Malik M. 1996. Heart rate variability: standards of measurement, physiological interpretation and clinical use. Task Force of the European Society of Cardiology and the North American Society of Pacing and Electrophysiology. Circulation 93:1043-65.

Macedo $M$ et al. 2008. Maternal wave reflections and arterial stiffness in normal pregnancy as assessed by applanation tonometry. Hypertension 51:1047-1051.

Mahendru A et al. 2014. A longitudinal study of maternal cardiovascular function from preconception to the postpartum period. J Hypertens 32: 849-856.

Malik M, Camm AJ. 1993. Components of heart rate variability - What they really mean and what we really measure. Am J Cardiol 72: 821-822.

Marañon R et al. 2014. Tempol blunts afferent arteriolar remodelling in chronic nitric oxide-deficient hypertension without normalizing blood pressure. Clinical and Experimental Hypertension 36: 132-139.

Nunan D, Sandercock G, Brodie D. 2010. A quantitative systematic review of normal values for short-term heart rate variability in healthy adults. PACE 33: 1407-1417.

Rakow A et al. 2013. Decreased heart rate variability in children born with low birth weight. Pediatric Research 74: 339-343.

Ramsay JE et al. 2003. Enhancement of Endothelial Function by Pregnancy. Diabetes Care 26(2): 475-479. 
Rodger M, Sheppard D, Gandara E, Tinmouth A. 2015. Haematological problems in obstetrics. Best Prac Res Clin Obstet Gynaecol 29: 671-684.

Sahni R et al.. Maturational changes in heart rate and heart rate variability in low birth weight infants. Dev Psychobiol 2000; 37: 73-81.

Savvidou M, Kaihura C, Anderson J, Nicolaides K. 2011. Maternal arterial stiffness in Women Who subsequently develop Pre-eclampsia. PloS ONE 6(5):e18703

Tanaka L et al. 2015. Exercise improves endothelial function: A local analysis of production of nitric oxide and reactive oxygen species. Nitric Oxide 15: 7-14.

Tarvainen M et al. 2014. Kubios HRV-heart rate variability analysis software. Comput Methods Programs Biomed 113: 210-220.

Toichi, M, Sugiera T, Murai T, Sengoku A. 1997. A new method of assessing cardiac autonomic function and its comparison with spectral analysis and coefficient of variation of R-R interval. Journal of the Autonomic Nervous System 62: 79-84.

Torrado $\mathrm{J}$ et al. 2015. Normal pregnancy is associated with changes in central hemodynamics and enhanced recruitable but not resting, endothelial function. Int $\mathrm{J}$ Reprod Med Volume 2015, Article ID 250951.

Vambergue A, Fajardy I. 2011. Consequences of gestational and pregestational diabetes on placental function and birth weight. World J Diabetes 15: 196-203.

Vieira de Oliveira G; Morgado M; Pierucci A; Silveira Alvares T. 2016. A single dose of a beetroot-based nutritional gel improves endothelial function in the elderly with cardiovascular risk factors. Journal of Functional Foods 26: 301-308

Walters WA, Lim YL. 1969. Cardiovascular dynamics in women receiving oral contraceptive therapy. Lancet 2:879-881

Williams $\mathrm{M}$ et al. 2001. Variations in endothelial function and arterial compliance during the menstrual cycle. J Clin Endocrinol Metab 86: 5389-5395. 
Wulsin L et al. 2015. Autonomic imbalance as a predictor of metabolic risks, cardiovascular disease, diabetes, and mortality. J Clin Endocrinol Metab 100: 24432448. 
Figure 1A

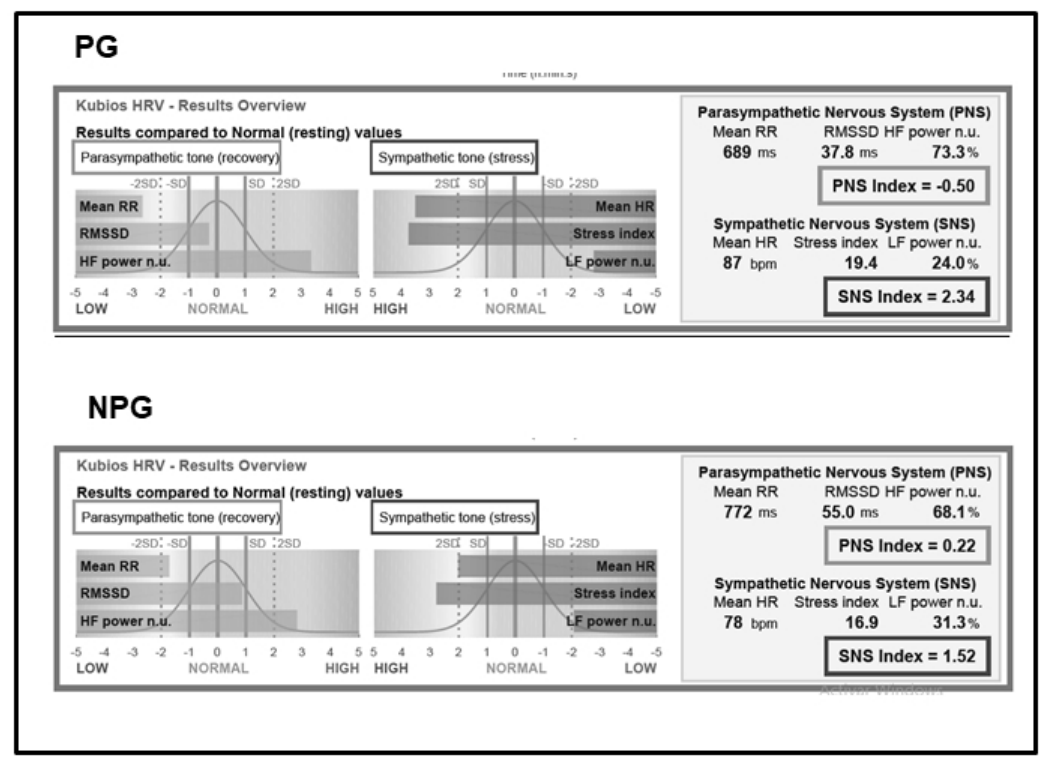

Figure 1B

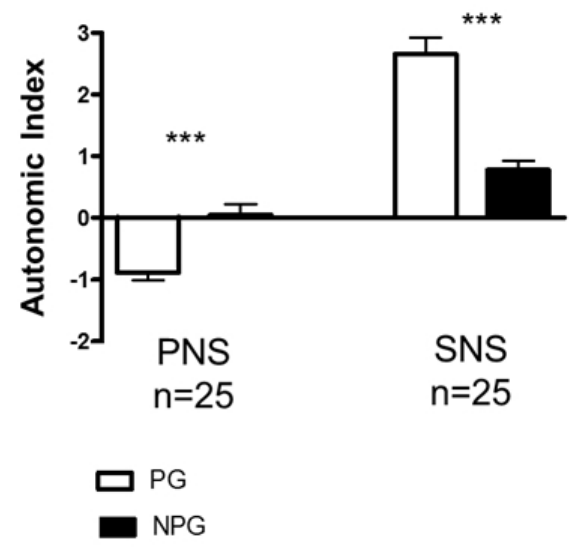

Figure 1: PNS and SNS indexes. Figure 1A: Typical report of Kubios HRV 3.1 of the variables used to computed PNS and SNS indexes in a woman in PG (top) and NPG (bottom). Figure 1B: Average of SNS and PNS indexes in the studied women. $* * *: \mathrm{p}<0.001$ PG vs NPG. Paired Student t-test. The data are expressed as the mean \pm standard error. The numbers of women tested are given in parentheses.

$$
60 \times 88 \mathrm{~mm}(300 \times 300 \mathrm{DPI})
$$


Figure 2A

Figure 2: Correlation between weight of newborns with the studied variables in PG. Figure 2A: Correlation with AS index; Pearson r: $-0.5004 ; 95 \% \mathrm{CI}$ : -0.7477 to $-0.1311 ; \mathrm{p}<0.05$. Figure 2B: Correlation with NOx; Pearson r: $0.5794 ; 95 \% \mathrm{CI}: 0.2388$ to $0.7930 ; \mathrm{p}<0.05$.

$60 \times 88 \mathrm{~mm}(300 \times 300 \mathrm{DPI})$ 
Figure 3A

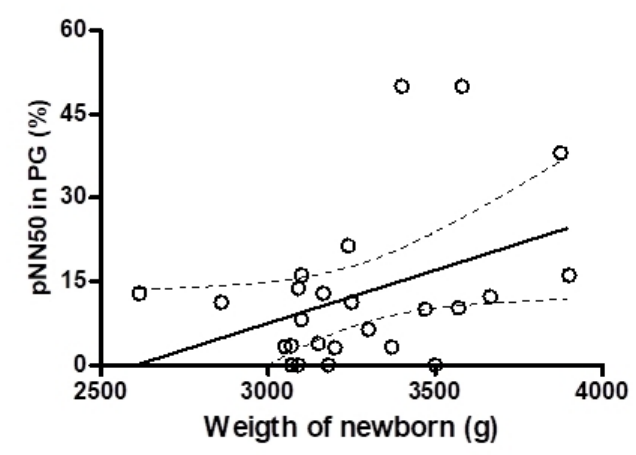

Figure 3B

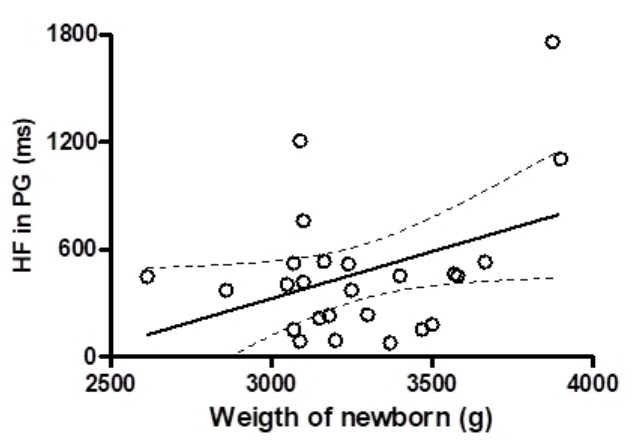

Figure 3C

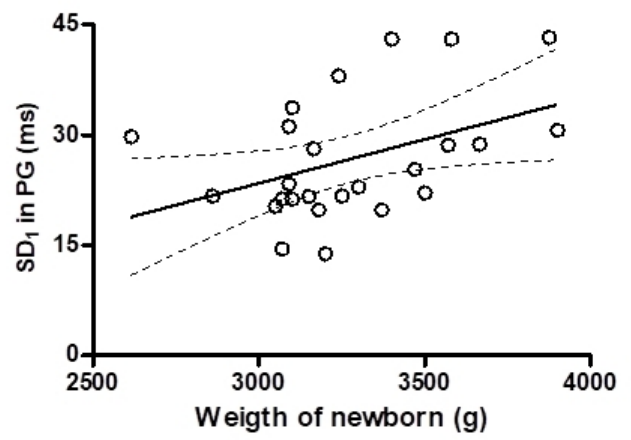

Figure 3: Correlation between weight of newborns with HRV in PG. Figure 3A: Correlation with pNN50; Pearson r: $0.4044 ; 95 \% \mathrm{CI}$ : 0.01098 to $0.6895 ; \mathrm{p}<0.05$. Figure 3B: Correlation with HF; Pearson r: 0.3999 ; 95\%CI: 0.005587 to $0.6866 ; p<0.05$. Figure 3C: Correlation with SD1; Pearson r: $0.4234 ; 95 \%$ CI: 0.03391 to $0.7013 ; \mathrm{p}<0.05$.

$60 \times 88 \mathrm{~mm}(300 \times 300 \mathrm{DPI})$ 


\section{TABLE 1}

Heart rate variability in the studied women $(n=25)$

\begin{tabular}{llll}
\hline Variable & Units & PG & NPG \\
\hline Time Domain & & & \\
HR & bpm & $90 \pm 2$ & $76 \pm 1 * * *$ \\
RR interval & ms & $687 \pm 17$ & $792 \pm 14 * * *$ \\
$\mathrm{pNN}_{50}$ & $\%$ & $13 \pm 3$ & $30 \pm 3 * * *$ \\
$\mathrm{SDNN}$ & $\mathrm{ms}$ & $33 \pm 2$ & $49 \pm 3 * * *$
\end{tabular}

Frequency Domain

$\begin{array}{lccc}\text { LF } & \mathrm{ms}^{2} & 397 \pm 94 & 1029 \pm 257^{*} \\ \text { HF } & \mathrm{ms}^{2} & 470 \pm 78 & 1312 \pm 381^{*} \\ \text { Total power } & \mathrm{ms}^{2} & 963 \pm 152 & 2530 \pm 596^{* *} \\ \text { LF/HF ratio } & \text { ratio } & 0.9 \pm 0.1 & 1.2 \pm 0.3 \mathrm{NS}\end{array}$

